\title{
A Platform as a Service for Smart Home
}

\author{
Boyun Eom, Choonhwa Lee, Changwoo Yoon, Hyunwoo Lee, and Won Ryu
}

\begin{abstract}
Owing to the convergence of home network, smart home technologies have been developing rapidly. However, all data generated from smart homes which might imply valuable information and be able to bring value-added outcome are not well utilized. Since it contains very private information, smart home users might want to avoid the chance to expose data from their living place. Moreover, few work concerns with how to deal with those valuable tentative but private data for further use. Motivated by this, we adapt cloud computing technology to smart home domain and design a framework which delivers a platform as a cloud computing service. The integrated framework we propose stores data generated from smart homes and provides these data for various services on the platform in a safer way to protect private data.
\end{abstract}

Index Terms - Cloud computing, PaaS, smart home.

\section{INTRODUCTION}

In recent years, cloud computing has been shaking up technology landscape. Besides reducing runtime, minimizing the risk of deploying physical infrastructure, and realizing green computing, perhaps, the most coveted feature would be the scalability that a cloud-based system brings forth. The system is flexible enough to expand, making it easier to deliver new services or applications. Users can access those services anytime anywhere as long as they can access Internet.

Smart home technologies have also developed continuously for more than a decade to improve the quality of life. Smart house is instrumented with various sensors and networking infrastructure and the major problem in this area is the integration and interaction among heterogeneous applications provided from different vendors. What in need are compelling services and value-added applications using data generated from smart homes as well as interoperability in smart home. Sensors at smart home are generating data which might imply valuable information almost every second, but its amount can be too much to be kept or to be analyzed at local side.

To solve these problems and to promote use of smart home service, we adapt smart home environment into cloud computing. Cloud computing provides abundant and powerful computing resources including storage, processors,

Manuscript received November 4, 2012; revised November 18, 2012. This work was supported by the IT R\&D program of MKE/KEIT. [10041673, Development of Hybrid Home Cloud Technology by Virtualization of Smart Home Appliance Resource]. This research was supported by the MKE(The Ministry of Knowledge Economy), Korea, under the ITRC(Information Technology Research Center) support program (NIPA-2012H0301-12-4003) supervised by the NIPA(National IT Industry Promotion Agency).

Choonhwa Lee is with Dept. of Computer Science and Engineering, Hanyang University, Seoul, Rep. of Korea (e-mail: lee@hanyang.ac.kr). and network connectivity [1].

Our primary approach is to move data generated from smart homes to the cloud side and provide these data to applications in a safe way. As a result, the architectural design of smart homes needs to be re-visited and modified accordingly.

The remainder of this paper is organized as follows; Section $\Pi$ reviews previous study closely related to our work. Section III discusses smart home requirements we have identified and in section IV, we present our design of a cloud-based smart home system. Section V explains the implementation of the prototyping system, and finally, section VI concludes this paper.

\section{RELATED WORK}

There have been several investigations to explore the potential for adopting cloud computing technologies to smart home domain.

Zhiqiang Wei and others proposed architecture of a smart home where smart home nodes and cloud servers form a peer-to-peer network [2].

Chang ho Yun has suggested cloud computing platform which get data from various kinds of remote sensors and remote video cameras and process those data with context-awareness [3]. As a middleware, this platform functions between various applications for ubiquitous city and infrastructure of u-city.

A Web 2.0 service platform for DPWS (Devices Profile for Web Services)-based home appliances were introduced for cloud environments [4]. The platform automatically records and observes user's behaviors to adapt home appliances to the changing need of the residents. By employing ontology technology, it can obtain more effective scalability, mobility, and interoperability.

Florian Daniel and others proposed integrating typical personal health record with environmental and sensor data and monitoring and analyzing an individual's habits so that individuals obtain life-style related advice to improve their health [5].

Other noteworthy efforts include a conceptual model to merge smart home into cloud [6] and computer-aided design software for smart home device via cloud service so that designers select smart home device and build living space [7].

As seen, several works have been studied for different purposes in various approaches in deploying smart home in cloud computing environment. However, little effort has been made to provide an integrated platform which provides data from smart spaces to value-added services in a safe and efficient way. 


\section{REQUIREMENTS OF ClOUd SMART HOME PlatFORM}

This section presents our architectural design for a smart home service platform. We discuss our design goal, policy, and a concern that we had in the course of the design process, first. And then we explain functional and non-functional requirements we identified

Providing smart home data to applications or users in a safe way is the key purpose of the platform we pursue. By nature, information gathered at home environments is very private. These personal data need to be stored and treated carefully not to be violated.

It is known that securing data might be the biggest challenge when deploying a system as a cloud computing service which shares computing resources across a network. In cloud computing environments where many service providers and systems are involved, security violations can occur often and easily. Therefore, storing personal data on cloud raises serious concerns about data protection. Cloud service users need an assurance of protecting from personal information abuse.

HEM (Home Energy Management) and HAS (Home Automation System) are traditional smart home services. Meanwhile, recently, home entertainment market has been heated up. Medical service at smart home is one of the promising areas in this field. By adapting smart home to cloud computing, more various services can be deliverable. Including the traditional services, roughly, we classify services on cloud-based smart home platform into two: services for controlling smart homes which were mostly conventional smart homes and value-added services. Value-added services are not so much for managing smart homes as for bringing an additional value or convenience to the home owners.

To make use of data for further processing, private data should often be shared with others or appropriate services. Being recognized as one of the most critical issues, security is thoroughly deliberated in our designing and added several components to deal with this. As part of the efforts, two important features are introduced onto our platform: delegation and encryption of sensitive information before stored.

Delegation is a process to expose data in a secure way to be shared. The owner of data can grant permission for data access to other users, other applications or other devices. Basically, not all data on the platform can be shared but only data whose owner allows to be accessed by others. For delegated data, sharing is possible only during the periods the owner specified for each device which generated data.

On the other hand, encryption is a process to keep data stored more securely. Since encryption and decryption can be expensive process, only those data regarded truly sensitive are encrypted.

Besides those two functional requirements, smart home platform should provide all functionalities of traditional smart homes as well as additional needs as a platform-as-a-service. There are three groups of users on cloud smart home platform: users of smart homes, service providers, and manufacturers of devices in smart home. Unlike other systems, in our platform, smart home service providers or developers are also one of user groups. We extracted each user group's requirements for cloud-based platform for each service and provide this in a matrix form as shown in Table I.

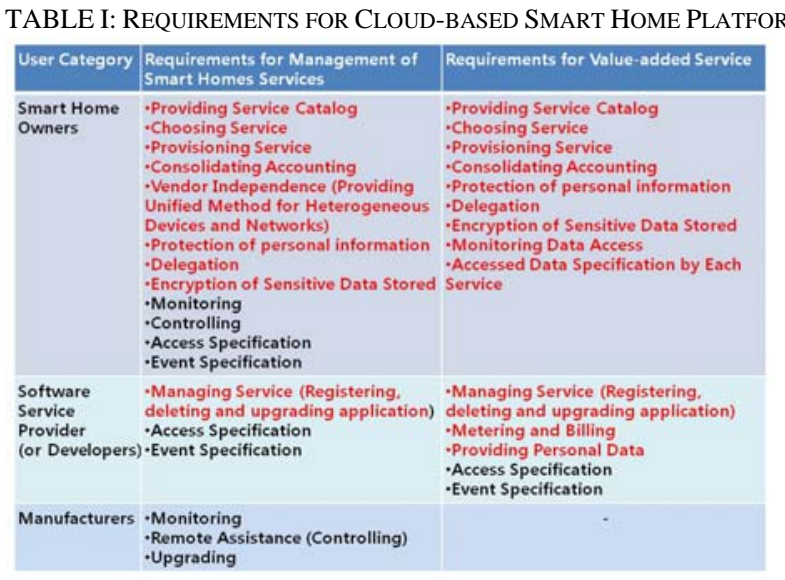

\section{Overall Architecture of SMART Home FRAMEWORK}

We designed the framework structure with components to meet the requirements we defined for each user classes and to accommodate cloud computing paradigm. There are four main parts in our framework: a real home gateway, a virtual home gateway, a smart home service platform, and smart screen devices. Clients might need only user interface to request a service to a cloud server, and thin or zero clients are easily found these days. Fig. 1 depicts overall structure of our framework.

\section{A. Real Home Gateway and Virtual Home Gateway}

The role of a typical home gateway is divided into two in our framework. A Real Home Gateway (RHG) plays a role of relaying data from home appliances and devices to the cloud. The other gate way, Virtual Home Gateway (VHG), on the cloud side takes care of rest roles of the conventional home gateway, as well as new additions.

In a cloud computing environment, computing resources are abundant and most operations are performed on servers on clouds, and upgrading software and provisioning service become easier. By our approach which integrates smart homes into cloud computing environments, a real home gateway or smart screen devices for the purpose of user interface can be a client which requests services to servers on clouds. Therefore, minimal functionalities like relaying data are left to a real home gateway in our framework design.

Once the Real Home Gateway receives data from home devices such as sensors, it sends these to a Virtual Home Gateway on cloud. A Virtual Home Gateway, then, parses received data and passes the results for making a decision on the sensitivity level of data. A VHG converts control-commands for each device when necessary. The converted commands are sent to a RHG and delivered to the device to control.

\section{B. Smart Home Platform}

Proposed smart home platform has a number of components to meet the requirements we defined: Personal/Home Data Manager, Device Manager, User 
Manager, Log Manager, Metering \& Billing Manager, Security Manager, and Service Manager.

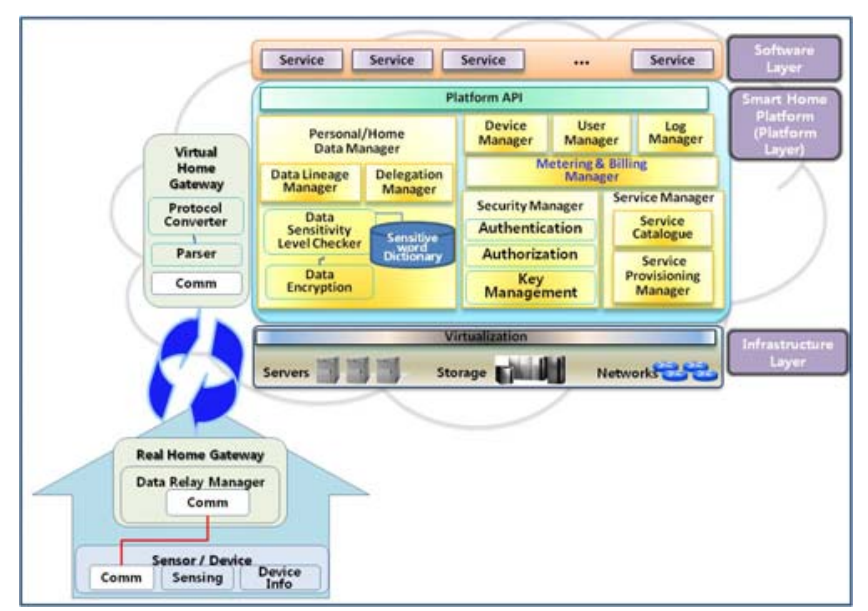

Fig. 1. Platform as a service for smart homes.

- Personal/Home Data Manager is a component for protecting personal information. It decides data sensitivity level by checking parsed data from a virtual home gateway. For the context-aware process, Personal/Home Data Manager uses a dictionary containing keywords for sensitivity and encrypts sensitive data before stored in cloud storage [8]. Fig. 2 is the flow of encryption. Data Lineage Manager in Personal/Home data manager watches activities towards stored data. It records every data access or history on data such that where it is from at what time, who accessed data, and when and where it is copied to. Monitoring data access, which is one of requirements from the smart home owners for value-added services, can be resolved by Data Lineage Manager. Delegation Manager is provided for delegation functionality. If smart home owners want to share certain data with others, or if they want to use a service which requires their personal data, then he or she can give permissions to others or other services.

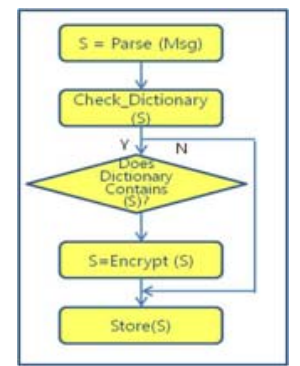

Fig. 2. Flow chart for storing sensed data.

- Device Manger creates virtual devices which are mapped to real devices at smart home and provides user interface for controlling and monitoring those devices. Moving operations for managing smart homes to cloud results in better flexibility in upgrading software, which is one of the prominent advantages in cloud computing service. It also manages device information such as device type, protocols, IP address, and etc.

- User Manager plays role for managing user profiles such as user identification, family members, and devices a user owns.

- Log Manager keeps track of events or activities over the system.

- Metering and Billing Manager is one of the indispensable components for cloud computing which is a blend of "utility computing". Smart home service platform integrates various services and the rate of a service can be varying. This component takes charge of service fees as well as the cost for cloud computing resource usage. It calculates total cost for smart home users who requested smart home services as well as service providers who consumed the platform for their service.

- Security Manager plays three roles: authentication, authorization, and key management. For all services on the platform, SSO (Single Sign On) might be convenient for users, which is also resolved with SPML(Service Provisioning Markup Language). Depending on the type of contents or service, different cryptosystem might be needed for key management.

- $\quad$ Service Manager: Service Catalogue in service manager provides a list with all possible services to smart home owners. Every service needs to register its functions. Provisioning service is also one of the requirements for value-added services and Service Provisioning Manager in service manager automates all the steps required to manage users or system access entitlements or data relative to electronically published service.

As illustrated in Fig. 1, smart home platform deploys onto cloud infrastructure where resources are typically virtualized. Platform API helps the developers write programs to run on our platform. Most requirements for value-added services from software service providers are provided through Platform API and characteristics of data or data types can be provided, as well.

\section{Smart Home User Interface: Smart Device}

In our framework, a real home gateway, a virtual home gateway, smart home user manager and device manager on smart home service platform, and smart home user interface take a part in performing the management functions for smart homes. Device Manager creates virtual devices which are mapped to real ones at smart homes and provides user interface to manage smart homes. Users can control, monitor, and make a schedule for those devices even outside the home through virtualized devices. When a user selects a control function of a certain device using the user interface, Device Manager creates a correspondent command and sends it to a VHG. As explained in the previous section, a VHG converts that command as for the proper protocol of the device.

\section{IMPLEMENTATION}

Most appliances connected to home networks have their own IP and communicate in IP-based protocols. In our implementation, we used two sensors and each sensor sends sensed data to a sink node through ZigBee protocol [9]. Every sensor device has a node id and communicates with a sink node which has the same group id. Using TCP/IP 
protocol, a sink node sends data from sensors to a real home gateway.

Fig. 3 illustrates the architecture of a prototyping system we implemented. We focused on delegation management in our prototyping system. The sensors we used are all for helping human health care: a biosensor which reads heart beats and a 3-axis motion sensor which calculates calories burned during human movements. These two sensors transmit sensed data to a sink node. We used a laptop connected to a sensor sink node via serial port and have it play the role as a RHG. Data sent from sensors are transmitted to a sink node which is connected to a laptop and the laptop sends these to a server on cloud side, VHG, through TCP/IP protocol. The VHG server runs a socket server as a daemon program and receives messages from the socket client on laptop (RHG).

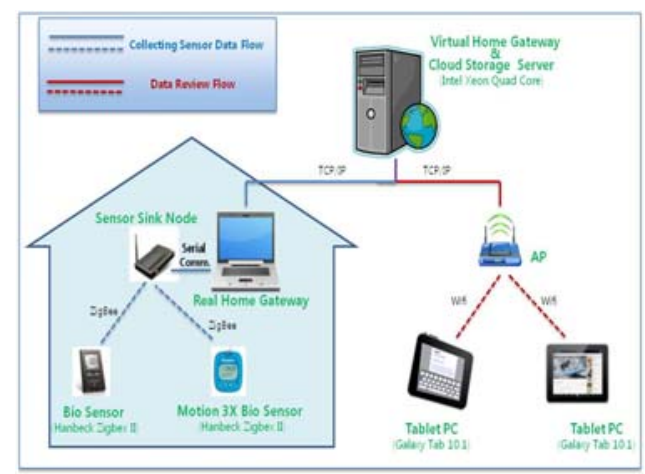

Fig. 3. Physical architecture of a prototype.

Typically, a home gateway is an embedded system but a personal computer with a sensor sink node acts as a Real Home Gateway in our implementation. Stored data are provided to the owner of data through smart screen device such as smart phones, smart pads, laptops or personal computers. We implemented user interface on Android platform for Tablets.

For the delegation management on proposed platform, at least two basic functions need to be implemented: assigning permission on data and revoking it from the grantee. In our implementation, users can check data and perform delegation for each device or each record during the periods he or she set. A user, user group or a service as a grantee also can be assigned by the owner.

Storing a huge amount of data from sensors and analyzing it can be expensive. Offloading this burden to a cloud makes it easier to introduce a new service that benefits from powerful computing resources on the network side. We provided analysis of daily, monthly or certain periodical calories burned or heart rates to the users in our implementation.

\section{CONCLUSION}

In this paper, we suggest a complete and integrated framework including a platform as a cloud computing service tailored for smart home environments. To organize functional needs for the framework, we classified services into two categories and summarized requirements for each service. We also design an overall architecture for smart home service platform comprised with several components to meet the requirements we identified for smart homes. Our concern was more about personal information protection while making data generated from smart homes available to the platform. We believe our approach which adapt smart home environment to cloud computing is effective to solve a couple of current smart home problems, and more smart home users or service providers employ the platform.

\section{REFERENCES}

[1] R. Buyyaa, C. S. Yeoa, S. Venugopala, J. Broberga, and I. Brandic, "Cloud computing and emerging IT platforms: Vision, hype, and reality for delivering computing as the 5th utility," Future Generation Computer Systems, vol. 25, no. 6, pp. 599-616, June 2009.

[2] Z. Wei, S. Qin, D. Jia, and Y. Yang, "Research and design of cloud architecture for smart home," in Proc. of International Conference on Software Engineering and Service Sciences, July 2010.

[3] C. Yun, H. Han, H. Jung, H. Yeom, and Y. Lee, “Intelligent management of remote facilities through a ubiquitous cloud middleware," in Proc. of 2009 IEEE International Conference on Cloud Computing, September 2009.

[4] C. Huang, "Design and implementation of a Web 2.0 service platform for DPWS-based home-appliances in the cloud environment," in Proc. of IEEE Workshop on Advanced Information Networking and Applications (WAINA), March 2011.

[5] F. Daniel, F. Casati, P. Silveira, M. Verga, and M. Nalin, "Beyond health tracking: A personal health and lifestyle platform," IEEE Internet Computing, vol. 15, no. 4, pp. 14-22, July-August 2011.

[6] Y. Yang, Z. Wei, D. Jia, Y. Cong, and R. Shan, "A cloud architecture based on smart home," in Proc. of the 2nd International Workshop on Education Technology and Computer Science (ETCS), March 2010.

[7] S. Chen and Y. Chang, "The computer-aided design software for smart home device based on cloud computing services,” in Proc. of the 2nd World Congress on Software Engineering (WCSE), December 2010.

[8] L. Nhang, Q. Wu, and Y. Hu, "Hierarchical identity-based encryption with constant-size private keys,” ETRI Journal, vol. 34, no. 1, pp. 142-145, February 2012.

[9] Z. Alliance. Zigbee. [Online]. Available: http://www.zigbee.org

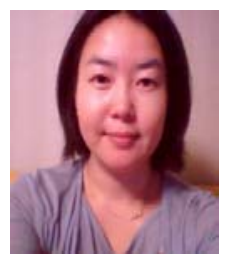

Boyun Eom was born in Cheongju in South Korea. She received her B.S. degree in computer engineering from Chungbuk National University, Cheongju, South Korea, in 1991 and M. S. degree in Computer \& Information Science \& Engineering from University of Florida, Gainesville, US, in 2005. Currently, she is a senior researcher at ETRI. Her research interests include cloud computing, smart home, database, and middleware and services computing technology.

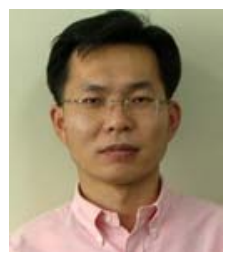

Choonhwa Lee received his B.S. and M.S. degrees in computer engineering from Seoul National University, South Korea, in 1990 and 1992, respectively, and his Ph.D. degree in computer engineering from the University of Florida, Gainesville, US, in 2003. He is an associate professor in the Division of Computer Science and Engineering at Hanyang University, Seoul, South Korea. Prof. Lee's research interests include mobile networking and computing, peer-to-peer networking protocols and systems, and middleware and services computing technology.

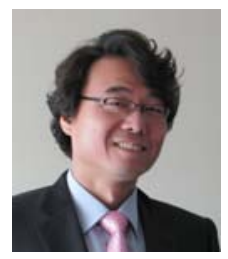

Changwoo Yoon received the B.S. degree from Sogang University, Seoul, Korea, in 1990. He received M. S. degree from POSTECH, Pohang, Korea, in 1992. He received Ph.D. degree in Computer \& Information Science \& Engineering from University of Florida, Gainesville, US, in 2005. Currently he is principal researcher and team leader in Virtual services platform team, ETRI and adjunct professor at UST. His current research interests include N-Screen, IPTV, Cloud computing, SOA, Service creation/delivery technology and information retrieval. 


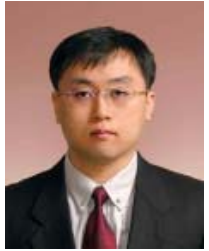

Hyunwoo Lee received M.S. and Ph.D. degrees in 1995 and 2005, respectively, in Korea Aerospace University (KAU). He is currently a principal research engineer and team leader in cloud convergence service networking research team, smart screen convergence research department, ETRI. His main research interests include heterogeneous wireless access network, Mobile P2P, open IPTV platform in NGN. His current research interests include cloud computing and platform.

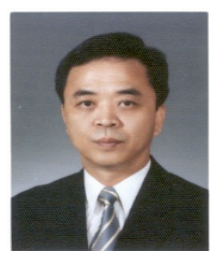

Won Ryu received the BS degree in computer science and statistics from Pusan National University, Busan, South Korea, in 1983, and the MS degree in computer science and statistics from Seoul National University, Seoul, South Korea, in 1987. He received his PhD degree in information engineering from Sungkyunkwan University, Kyonggi, South Korea, in 2000. Since 1989, he has been a managing director with the Smart screen convergence research department, ETRI, Daejeon, Korea. Currently, his research interests are IPTV, Smart TV, IMT-advanced, and convergence services and networks and etc. 